\title{
P-Wave Related Disease Detection Using DWT
}

\author{
Syeda Adiba Rehaman ${ }^{1,}$ P. Rajeswari ${ }^{2}$ \\ ${ }^{I}$ Final year M.Tech BMI, Dept. of IT, SJCE, Mysuru \\ ${ }^{2}$ Assistant Professor, Dept. of IT, SJCE, Mysuru
}

\begin{abstract}
ECG conveys information regarding the electrical function of the heart, by altering the shape of its constituent waves, namely the P, QRS, and T waves. ECG Feature Extraction plays a significant role in diagnosing most of the cardiac diseases. This paper focuses on detection of the P-wave, based on 12 lead standard ECG, which will be applied to the detection of patients prone to diseases. The ECG signal contains noise and that noise is removed using Bandpass filter. After elimination of noise, we detect QRS complex which help in detecting the P-Wave. P-wave morphology can be determined in leads II as monophasic and VI as biphasic during sinus rhythm. DWT provides a value that helps in estimating features of the P-Wave. This detects the diseases that occur when the P-wave is abnormal. The method has been validated using ECG recordings of 250 patients with a wide variety of $P$-wave morphologies from Database.
\end{abstract}

Keywords: Electrocardiogram, $P$ wave, Wavelet Transform, Discrete Wavelet Transform

\section{Introduction}

An ECG machine interprets and records the electrical impulses of the heart for diagnostic purposes. It is not a form of treatment for heart conditions. The Electrocardiogram is the contraction and relaxation of heart muscles generated when the atria and ventricles are repolarized and depolarized. It varies from person to person depending on the age, body weight position and various other factors that gives us information about persons heart rate, rhythm etc. ECG is important since heart diseases constitute one of the major causes of mortality in the world. The ECG is characterized by a sequence of P, QRS and T wave associated with each beat as shown in fig.1.

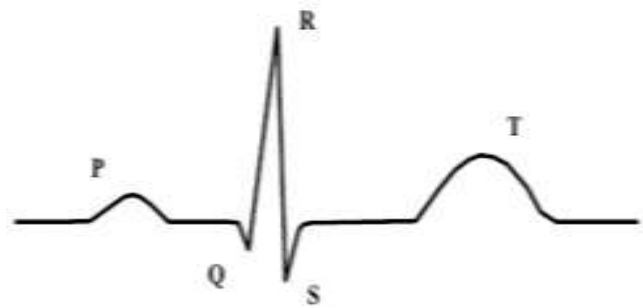

Fig. 1. An ECG waveform

The P wave is always before QRS complex and is separated by PQ interval. It is caused by atrial depolarization. The duration of P-wave is $110 \mathrm{~ms}$ and the positive wave always is at the lead II whereas negative is always at VR lead. The wavelet transform based technique can be used to identify the characteristic features of the ECG signal to obtain reasonably good accuracy with the presence of high frequency and low frequency noises. A number of techniques have been used by researchers to detect the characteristics in ECG [1]. We use dyadic wavelet transform approach because of its localization properties and fast calculations.

The Wavelet Transform decomposes signal into family of wavelets. Wavelets can be symmetric or asymmetric in shape. Recently, wavelets have been applied to numerous problems in Electrocardiology, including data compression, analysis of ventricular late potentials, and the detection of ECG characteristic points. The WT for evaluating higher frequencies and for lower frequencies uses a short and long time interval respectively.

\section{Methodology}

In order to extract information from the ECG signal, the raw ECG signal should be processed. ECG signal processing can be roughly divided into preprocessing, detection of P-wave and feature extraction as shown in Fig.2. 


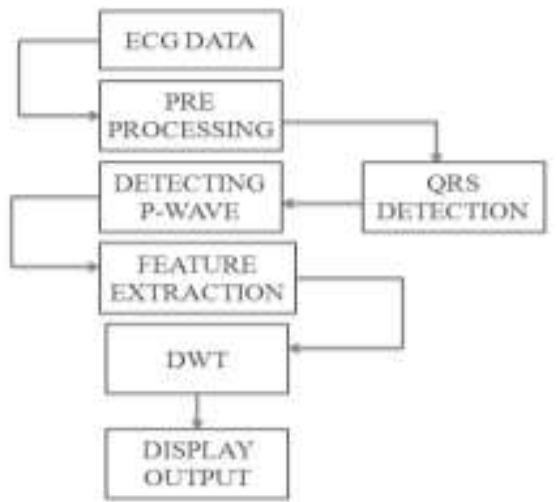

Fig.2. Structure of ECG Signal Processing

The ECG signal is collected from JSS Hospital, Mysuru. The signal is read using EDFbrower software. The data is preprocessed and features are extracted. A Feature Extraction method using Discrete Wavelet Transform (DWT) was proposed by Emran et al [2]. DWT is applied on the features extracted and we obtain wavelet co-efficients. There are four wavelet co-efficients and we select the co-efficient that gives values related to Pan-Tompkins algorithm. From the co-efficients we get maximum and minimum values which are used to locate P-wave. Once we achieve P-wave, we check for the diseases involved that is if the P-wave is abnormal.

\subsection{Pre-processing}

ECG signal mainly contains noise.The purpose of preprocessing is to remove artifacts from raw signal. Artifacts are the electrical interference by outside sources, electrical noise,baseline wandering muscle interference etc. The two main problems in ECG signal processing is baseline wander removal and suppression of noise.Baseline wandering is one of the noise artifacts that affect ECG signals. We use the median filters[3] to eliminate baseline drift of ECG signal. To remove the noise, we use Discrete Wavelet Transform.

\subsection{Detection of QRS Complex}

QRS detection is difficult, because of the various types of noise that can be present in the ECG signal. Noise sources include muscle noise, artifacts due to electrode motion, power-line interference, baseline wander, and $\mathrm{T}$ waves with high-frequency characteristics similar to QRS complexes. Here the filters reduce noise and thereby increase signal to noise ratio[4]. Software QRS detectors typically include three different types of processing steps: linear digital filtering, nonlinear transformation, and decision rule algorithms. We used linear digital filtering which uses bandpass filter for elimination of noise.

\subsection{Detection of P-Wave}

The ECG signal obtained is contaminated generally by different sources of noises that can disrupt the phase and the amplitude characteristics of the useful signal, where there is necessity of a good filtering [5]. We apply a band pass filter [6] to eliminate the noises caused by the breathing, the movements of muscles and the baseline[7].

In general, the normal ECG means that there is a regular normal rhythm and waveform. However, the ECG rhythm of the patient with arrhythmia will not be regular in certain QRS complexes. Therefore, we find the characteristics that help us to obtain a perfect ECG waveform. We first need to find the location of every QRS complex. The locations of QRS complex have the maximum variation in the slopes. The method of PanTompkins was adopted to detect the QRS complex [8]. The Pan and Tompkins QRS detection algorithm identifies the QRS complexes based upon digital analysis of slope, amplitude, and width of the ECG data. The algorithm implements a special digital band pass filter. It can reduce false detection caused by the various types of interference present in the ECG signal. Once we get the location of QRS complex, P-wave can be located easily by getting the maximum and minimum values which denote a P-wave. After obtaining a noise free Pwave, we check for its normality. In some cases, the P-wave is absent. Hence, we detect the diseases that occur when there is abnormal P-wave. The diseases being detected are atrial fibrillation, atrial flutter etc.

\subsection{Feature Extraction and DWT}

The Feature Extraction stage extracts diagnostic information from the ECG signal. It provides us the features extracted using DWT such as the co-efficient obtained after passing through filters and being downsampled produce approximate, horizontal, diagonal and vertical co-efficients respectively. 
Discrete Wavelet Transform (DWT) can be used as a good tool for analyzing non-stationary ECG signal as wavelet transform has been proven to be useful tool for non-stationary signal analysis. The DWT of a signal is calculated by passing it through a series of filters as in fig.3, first the samples are passed through a low pass filter with impulse response $\mathrm{g}$ (Eq.1) and then it is passed through high pass filter $\mathrm{h}$ (Eq.2). The output gives wavelet coefficient values $1^{\text {st }}, 2^{\text {nd }}$ etc represent approximate, diagonal, vertical and horizontal respectively.

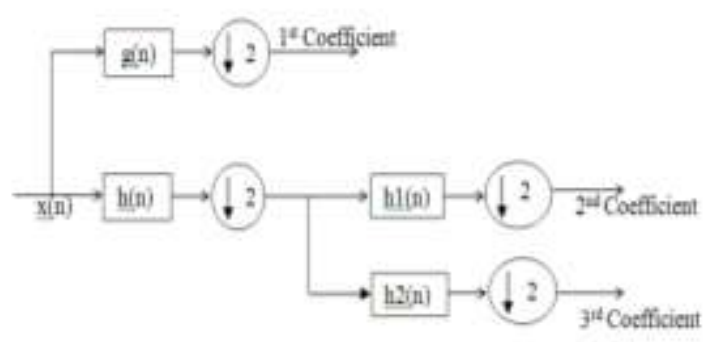

Fig. 3. Block diagram of DWT filter analysis

However, since half of the frequencies are removed the other half are discarded using Nyquists rule. The filter output of the low-pass filter $h$ is then subsampled by 2 and further processed by passing it again through a new low-pass filter h1 and a high-pass filter h2 with half the cut-off frequency of the previous one, i.e:-

$$
\begin{array}{r}
\text { ylow }[\mathrm{n}]=\sum_{k=-\infty}^{\infty} x[k] g[2 n-k] \\
\operatorname{yhigh}[\mathrm{n}]=\sum_{k=-\infty}^{\infty} x[k] h[2 n-k]
\end{array}
$$

\begin{tabular}{|l|l|l|l|l|l|l|}
\hline Co-efficients & \multicolumn{6}{|l|}{ Co-efficients value } \\
\hline Approximate & 157 & 178 & 197 & 213 & 162 & 236 \\
\hline Horizontal & 0.0 & 0.0 & 0.0 & 0.0 & 0.0 & 0.0 \\
\hline Diagonal & -9 & -12 & 3 & 19 & -70 & 4 \\
\hline Vertical & 0.0 & 0.0 & 0.0 & 0.0 & 0.0 & 0.0 \\
\hline
\end{tabular}

Table I: Co-efficient Values.

The coefficient values listed in Table I are obtained after applying DWT to the P-wave that was detected after elimination of noise.

The co-efficient values in the table I show values for Approximate, Horizontal, Vertical and Diagonal and the Pwave can be plotted using approximate and diagonal values since horizontal and vertical co-efficient values show zero(0) result because it contains noise that is eliminated.
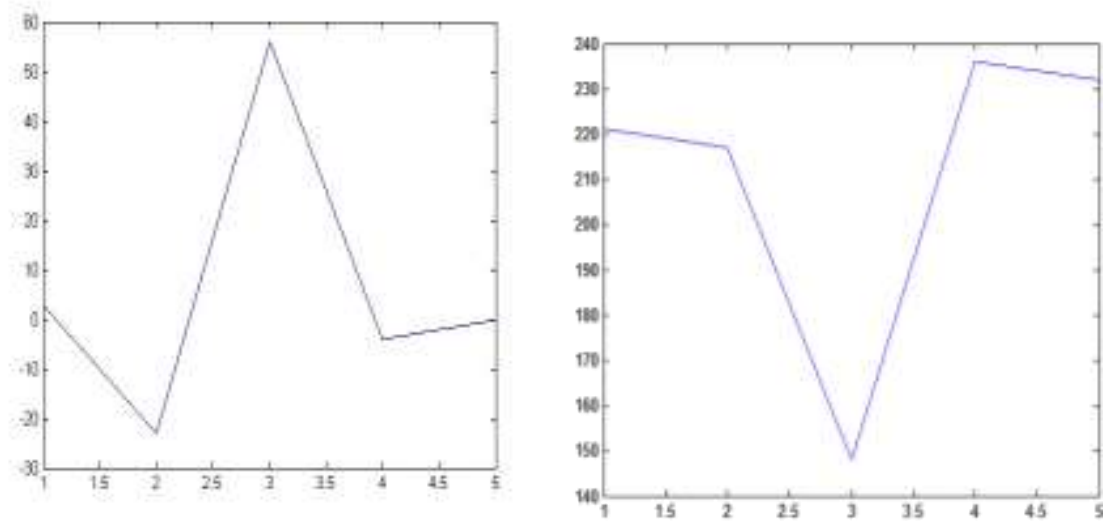

Fig. 4 (a). P-wave plot with diagonal co-efficient value obtained after applying DWT and (b). P-wave plot with approximate co-efficient value obtained after applying DWT where X-axis represents co-efficient values and Yaxis represents amplitude. 


\section{Result And Conclusion}

ECG signals required for analysis are collected from JSS Hospital, Mysuru. The database contains 250 records. The methods were developed under Matlab software.

In this paper, we present an algorithm based on Wavelet Transform for the detection of P waves of ECG. ECG characteristic points based on WT shows the potential of the WT especially for processing time-varying biomedical signals. The power of WT lies in its multiscale information analysis which can characterize a signal very well. It is clear that the WT method will lead to a new way of biomedical signal processing.

A total of 40 data sets where collected out of which 30 were diseased which had atrial fibrillation, atrial flutter, sinus rhythm and 10 were normal ECG. The algorithm has correctly classified sinus rhythm and normal set whereas the atrial fibrillation and atrial flutter have false estimation as shown in Table II.

\begin{tabular}{|l|l|l|l|}
\hline Disease & $\begin{array}{l}\text { Number of } \\
\text { Samples }\end{array}$ & $\begin{array}{l}\text { True } \\
\text { Estimation }\end{array}$ & False Estimation \\
\hline Atrial Fibrillation & 10 & 07 & 03 \\
\hline Atrial Flutter & 10 & 09 & 01 \\
\hline Sinus Rhythm & 10 & 10 & 00 \\
\hline Normal & 10 & 10 & 00 \\
\hline
\end{tabular}

Table II: Displaying disease

The P-wave detected checks the range of the disease and converts into frequency value and gives a normal signal or diseased name as an output.

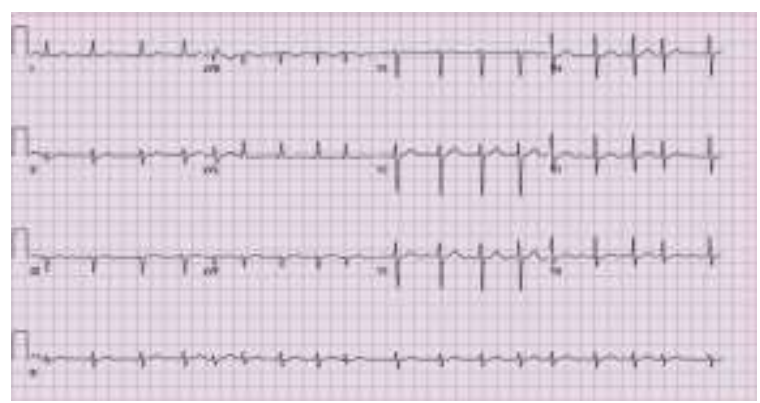

Fig.5 (a)Atrial Fibrillation

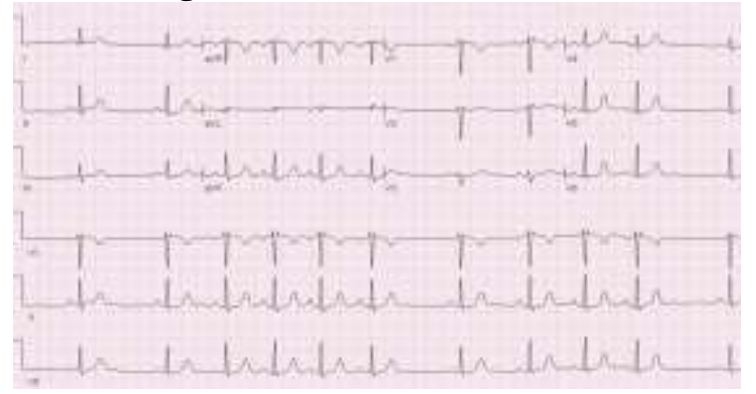

Fig.5 (c) Sinus Arrhythmia

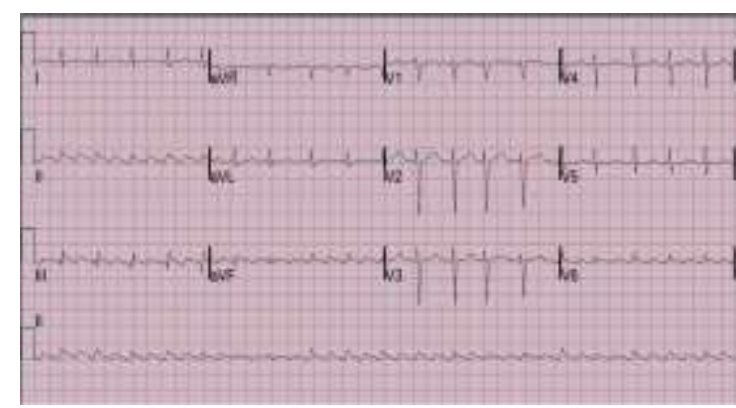

Fig.5 (b) Atrial Flutter

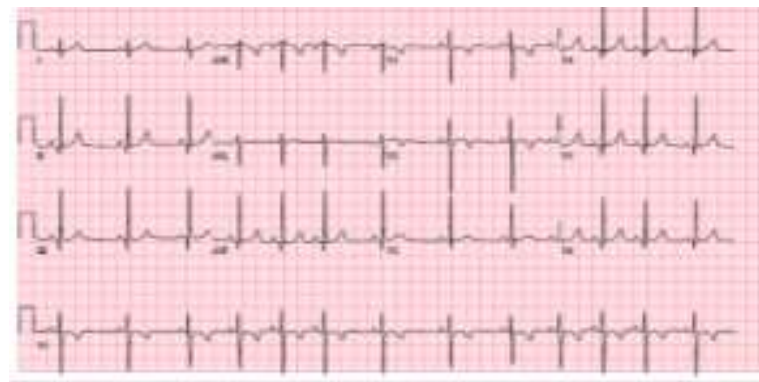

Fig.5 (d) Sinus Rhythm/ Normal

The diseases related to P-wave such as Atrial Fibrillation, Atrial Flutter, Sinus Arrhythmia and Sinus Rhythm/ Normal are shown in fig 5 (a), (b), (c)and (d) respectively.

\section{Acknowledgement}

It gives great pleasure in presenting the paper on "P-Wave Related Disease Detection Using DWT". We would like to take this opportunity to thank JSS Hospital, Mysuru for providing ECG data of the patients. We are also grateful to our Department of Instrumentation, SJCE, Mysuru for their indispensable support and suggestions. 


\section{References}

[1]. J. Pan and W. J. Tompkins, “A real-time QRS detection algorithm”, IEEE Trans. Biomed. Eng., vol. 32, pp. 230-236, 1985.

[2]. http://dx.doi.org/10.1109/TBME.1985.325532

[3]. E. M. Tamil, N. H. Kamarudin, R. Salleh, M. Yamani Idna Idris, M. N. Noorzaily, and A. M. Tamil, (2008) "Heartbeat electrocardiogram

[4]. (ECG) signal feature extraction using discrete wavelet transforms (DWT)", in Proceedings of CSPA, 1112-1117.

[5]. Saritha, V. Sukanya, and Y. Narasimha Murthy, "ECG Signal Analysis Using Wavelet Transforms", Bulgarian Journal of Physics, vol. 35, pp. 68-77, 2008.

[6]. 0. Pahlm and L. Sornmo, "Software QRS detection in ambulatory monitoring-A review," Med. Biol. Eng. Comput., vol. 22, pp. 289-297, 1984.

[7]. S. Z. Mahmoodabadi, A. Ahmadian, and M. D. Abolhasani, "ECG Feature Extraction using Daubechies Wavelets", Proceedings of the fifth IASTED International conference on Visualization, Imaging and Image Processing, pp. 343-348, 2005.

[8]. McNames, J. (2005) Optimal Rate Filters for Biomedical Point Processes. 27th Annual International Conference of the Engineering in Medicine and Biology Society, Shanghai, 17-18 January 2006, 145-148. http://dx.doi.org/10.1109/iembs.2005.1616363.

[9]. Li, C., Zheng, C. and Tai, C. (1995) Detection of ECG Characteristic Points Using Wavelet Transform. IEEE Transactions on Biomedical Engineering, 42, 21-28. http://dx.doi.org/10.1109/10.362922

[10]. Khelil, B., Kachouri, A. and Ben Messaoud, M. (2006) Nouvelle Tendance Technologiques en Génie Electrique et Informatique. Springer, New York.

[11]. Lee, J., Jeong, K., Yoon, J. and Lee, M., “A Simple Real-Time QRS Detection Algorithm”, 18th Annual International Conference of the IEEE Engineering in Medicine and Biology Society, Amsterdam 1996.

[12]. J.S. Sahambi, S.N. Tandon, R.K.P. Bhatt, "Using wavelet transforms for ECG characterization”, IEEE Eng. Med. Biol., vol. 16, no. 1 , pp. 77-83, 1997.

[13]. Di Virgilio, V., Francalancia, C., Lino, S., Cerutti, S., "ECG Fiducial Points Detection Through Wavelet Transform”, 17th Annual International Conference of the IEEE Engineering in Medicine and Biology Society and CMBEC, 1995, Theme 4: Signal Processing. 